\title{
Two-dimensional electronic spectroscopy of graphene nanoribbons in organic solution
}

\author{
Tetsuhiko Nagahara ${ }^{1,2, *}$, Lucia Ganzer $^{1}$, Franco V. A. Camargo ${ }^{1}$, Yinjuan Huang ${ }^{3}$, Fugui Xu ${ }^{3}$, \\ Yiyong $\mathrm{Mai}^{3}$, Giulio Cerullo ${ }^{1}$, and Xinliang Feng ${ }^{4}$ \\ ${ }^{1}$ IFN-CNR, Dipartimento di Fisica, Politecnico di Milano, Piazza L. da Vinci 32, 20133 Milano, Italy \\ ${ }^{2}$ Department of Chemistry and Materials Technology, Kyoto Institute of Technology, Matsugasaki, \\ Sakyo-ku, 606-8585 Kyoto, Japan \\ ${ }^{3}$ School of Chemistry and Chemical Engineering, Shanghai Jiao Tong University, 800 Dongchuan Road, \\ Shanghai 200240, China \\ ${ }^{4}$ Department of Chemistry and Food Chemistry, Technische Universität Dresden, Mommsenstrasse 4, \\ 01062 Dresden, Germany
}

\begin{abstract}
We unravel the electronic structure of graphene nanoribbons in solution using two-dimensional electronic spectroscopy. We identify different excitons, their vibrational couplings and find that exciton diffusion or relaxation among the exiton sublevels takes place in $\sim 300 \mathrm{fs}$.
\end{abstract}

\section{Introduction}

Graphene nanoribbons (GNRs) are nanometer-wide graphene structures that, unlike graphene itself, possess a bandgap due to quantum confinement along the ribbon width. Their optical, electronic and magnetic properties are all controllable by the width and edge structures of GNRs, making them excellent candidates for next generation semiconducting materials [1]. On the other hand, strong $\pi-\pi$ interactions between GNRs lead to the formation of aggregates with different properties than the intrinsic ones. Chemically synthesized GNRs dispersed in organic solvents have been reported, but spectroscopic evidence of substantial aggregation remained present $[2,3]$.

Very recently, GNRs with armchair-edge structure with 1-1.7 nm width and variable lengths up to $60 \mathrm{~nm}$ were synthesized (Fig. 1). Thanks to the addition of bulky side groups very high solubility was achieved and steady state absorption, photoluminescence (PL) as well as transient absorption (TA) spectroscopies suggest that the solutions consist mainly of isolated single ribbons, even for lengths of $60 \mathrm{~nm}$ [4]. The linear absorption spectra of the GNRs in toluene (Fig. 1) are very broad and yet structured, suggesting that a rich electronic structure might be present.

Here we employ two-dimensional electronic spectroscopy (2DES) to study the electronic structure and dynamics of the $60 \mathrm{~nm}$ long GNR in solution. The 2DES setup is based on the partially collinear pump-probe geometry using a birefringent interferometer for the generation of the phase-locked pump pulse pair and employs $\approx 10 \mathrm{fs}$ broadband pulses generated with visible (VIS, 530-730 nm) and near infrared (NIR, 650-1050 nm) non-collinear OPAs $[5,6]$.

\footnotetext{
*e-mail: nagahara@kit.ac.jp
} 

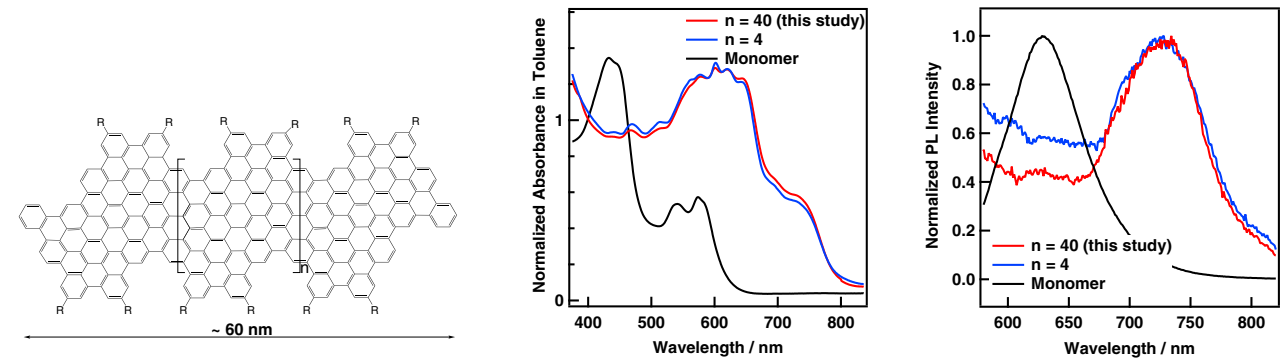

Figure 1. Molecular structure of the GNR and its absorption and PL spectra in toluene. The spectra are independent of the length while redshifted from the monomer. The absorption spectrum shows a convoluted structure of peaks, while the lowest main exciton peak $(735 \mathrm{~nm})$ is identified in the PL.

\section{Results and discussion}

The 2DES maps (Fig. 2) reveal that the broad absorption spectrum of the GNR consists of a series of many sharp peaks. In NIR region, we identify a diagonally stretched signal extending from 690 to $760 \mathrm{~nm}$ with intense exciton peak at $735 \mathrm{~nm}$. There is an off-diagonal cross peak between 690 and $760 \mathrm{~nm}$ both of which cannot be assigned to vibration coupled transitions of the intense main lowest energy exciton at $735 \mathrm{~nm}$, as there are no off-diagonal peaks connecting them to $735 \mathrm{~nm}$ to prove any coupling. From excitation wavelength dependent PL measurements, we also identify a relatively weak emission peak at $760 \mathrm{~nm}$ by 690 and $760 \mathrm{~nm}$ excitations. Thus the diagonally stretched feature is actually consisting of two exciton species: an intense main exciton at $735 \mathrm{~nm}$ and a relatively weak one at 690 and 760 $\mathrm{nm}$. The weak exciton species is likely to be conformationally different from the main one.

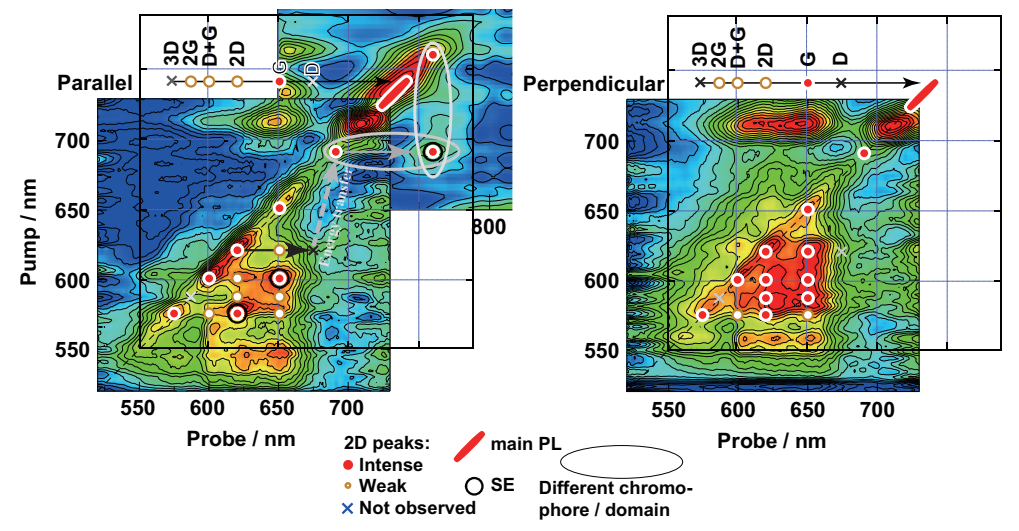

Figure 2. 2DES maps of GNR in VIS and NIR at $t_{2}=1 \mathrm{ps}$ for parallel and perpendicular polarization combinations. At $735 \mathrm{~nm}$, which exactly matches the PL peak in Fig. 1, the lowest main exciton peak is seen. The main exciton transition coupled to vibrational modes of $D, G, 2 D, D+G, 2 G$ and $3 D$ form these mesh grid-like structures. The diagonal peaks at $690,760 \mathrm{~nm}$ and their cross peak are assigned to a different exciton species.

The VIS 2DES maps reveal various diagonal and off-diagonal peaks that indicate strong coupling between the main exciton at $735 \mathrm{~nm}$ and Raman active vibrational modes $(\mathrm{D}=1320$, $\mathrm{G}=1610 \mathrm{~cm}^{-1}$ and their combinations) of graphene. Computing the expected separation 
between different Raman modes and the main exciton at $735 \mathrm{~nm}$, we find that the mesh gridlike structure shown in VIS is well described by vibronic coupling between the main exciton and vibrational modes which are also observed by resonance Raman. From polarization dependence, we found that intense off-diagonal peaks at $3 \mathrm{D} \rightarrow 2 \mathrm{D}, \mathrm{D}+\mathrm{G} \rightarrow \mathrm{G}$ of the main exciton and $690 \mathrm{~nm} \rightarrow 760 \mathrm{~nm}$ of the weak exciton are stimulated emissions (SE), all of them shifted by energy of D mode from excitations. Broadband TA measurements featured intense oscillatory amplitude mainly coming from the D mode, further confirming the strong vibrational coupling. In connection, Raman D band intensities of GNRs are known to be sensitive to the widths, edge structures and positions of side groups that determine the exciton transitions [7]. Analogous 2D $\rightarrow$ D SE peak would be also expected. However, the lack of this SE cross peak is explained by efficient energy transfer to the nearby state of weak exciton species at $690 \mathrm{~nm}$, which is also confirmed by PL measurements.

The dynamic behaviours of different coordinates in the 2DES maps (Fig. 3) show that all diagonal and the off-diagonal SE peaks present a $\sim 300 \mathrm{fs}$ decay, in contrast to the other offdiagonal peaks (only bleach and some eventual overlapping excited state absorption). This partial decay of the SE signals is likely due to diffusion of the excitons or relaxation among the intraexcitonic sublevels which is also suggested in single wall carbon nanotube [8].
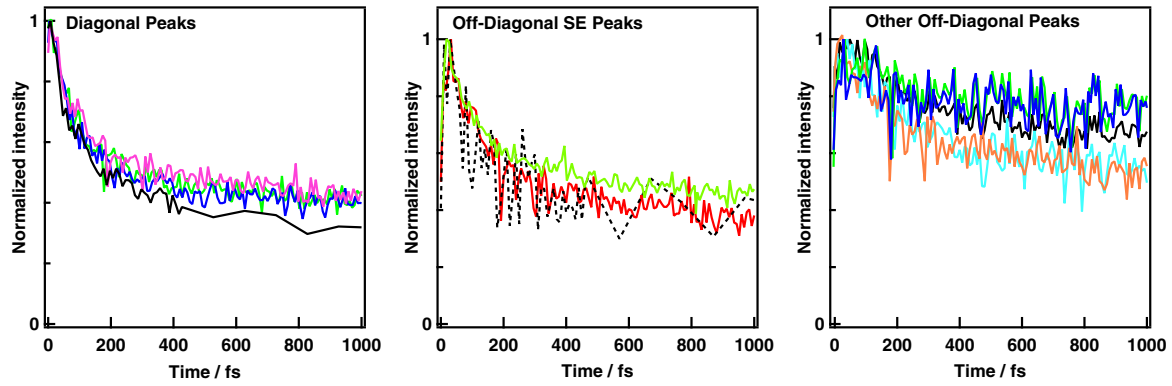

Figure 3. The fast $\approx 300 \mathrm{fs}$ decays shown in diagonal (left) and off-diagonal stimulated emission peaks (middle) suggest exciton diffusion or intraexcitonic relaxation.

In summary, VIS and NIR 2DES were used to unravel the excited state structure of highly soluble GNRs with $60 \mathrm{~nm}$ length and 1-1.7 nm width in toluene solution, revealing the presence of two different exciton species and strong vibrational coupling between the excitons and the Raman active vibrational modes of graphene. Finally, we observed exciton diffusion or intraexcitonic relaxation on the $\sim 300$ fs timescale.

\section{References}

[1] M. Ezawa, Phys. Rev. B. 73, 045432 (2006)

[2] Y. Huang et al., J. Am. Chem. Soc. 138, 10136 (2016)

[3] I. Ivanov et al., J. Am. Chem. Soc. 139, 7982 (2017)

[4] Y. Mai et al., J. Am. Chem. Soc. , submitted

[5] J. Réhault et al., Rev. Sci. Instrum. 85, 123107 (2014)

[6] D. Brida et al., J. Opt. 12, 013001 (2009)

[7] M. G. Schwab et al., J. Am. Chem. Soc. 134, 18169 (2012)

[8] X. Xu et al., J. Phys. Chem. C 113, 18106 (2009) 